\title{
High-Resolution Spectroscopy of some Active Southern Stars
}

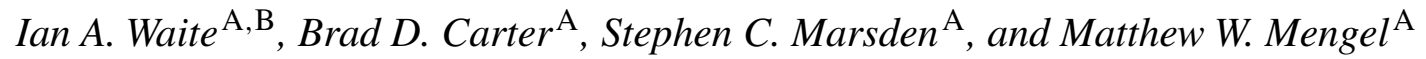 \\ ${ }^{A}$ Centre for Astronomy, Solar Radiation and Climate (CASRAC), University of Southern Queensland, \\ Toowoomba QLD 4350, Australia \\ ${ }^{\text {B } C o r r e s p o n d i n g ~ a u t h o r . ~ E m a i l: ~ w a i t e @ ~ u s q . e d u . a u ~}$
}

Received 2004 May 13, accepted 2004 September 27

\begin{abstract}
High-resolution échelle spectra of 42 nearby southern solar-type stars have been obtained, in a search for young, single, active, and rapidly rotating sun-like stars suitable for Doppler imaging and Zeeman Doppler imaging studies. As a result of this survey, 13 stars were determined to be youthful with ages less than $600 \mathrm{Myr}$ (Hyades age) and eight of these were found to have projected rotational velocities in excess of $15 \mathrm{~km} \mathrm{~s}^{-1}$. In addition, five spectroscopic binary systems were identified. Of those stars observed for this survey, HD 106506 is the most outstanding target for mapping active regions. It is an apparently young and single star with rapid rotation $\left(v \sin i \sim 80 \mathrm{~km} \mathrm{~s}^{-1}\right.$ ), strong $\mathrm{H} \alpha$ chromospheric activity (log $\left.R_{\mathrm{H} \alpha}^{\prime} \sim-4.2\right)$, and deformation of the spectral line profiles indicating the presence of large starspots.
\end{abstract}

Keywords: stars: activity — stars: spots — stars: magnetic fields — stars: rotation

\section{Introduction}

Young solar-type F-, G-, and K-stars provide proxies for the early Sun, and insight into its intense zero-age main sequence magnetic activity. In this regard, observations of starspots and surface magnetic fields are particularly important, as they provide the empirical basis for understanding the star's magnetic dynamo and its internal structure. A technique to define the location of starspots and hence map the surface of stars is known as Doppler imaging (DI). Fekel (1980) observed that the distortions in the line profiles of stars appeared to be correlated with the orbital phase. Vogt \& Penrod (1983) developed DI as a way of deriving a map of the surface of the star. They specify the minimum projected rotational velocity $(v \sin i)$ for this technique to be roughly $25-30 \mathrm{~km} \mathrm{~s}^{-1}$ with a signal to noise $(\mathrm{S} / \mathrm{N})$ of approximately 200 . Nevertheless, Strassmeier \& Rice (1998) have used DI to produce a map of the surface of EK Draconis, a star with a projected rotational velocity of only $17.3 \mathrm{~km} \mathrm{~s}^{-1}$. On the other hand, the most rapidly rotating star that has been successfully mapped using DI has been the ultra-fast rotator VXR45a, with a $v \sin i$ of $238 \mathrm{~km} \mathrm{~s}^{-1}$, in the young open cluster IC2391 (Marsden et al. 2004).

Starspots, like sunspots, are caused by magnetic fields. However, the strength and distribution of the magnetic fields of solar-type stars are notoriously difficult to measure. For young and active rapidly rotating stars $\left(v \sin i \gtrsim 25 \mathrm{~km} \mathrm{~s}^{-1}\right)$, we can use a spectropolarimetric technique called Zeeman Doppler imaging (ZDI; Donati et al. 1989; Semel 1989; Donati et al. 2003). ZDI uses the Semel spectropolarimeter, or SEMPOL (Semel et al. 1993; Donati et al. 2003), and is a visitor instrument on the 3.9-m Anglo-Australian Telescope (AAT). The Zeeman signature in the spectra of solar-type stars is tiny, with a typical polarization of $0.1 \%$ for active stars (Donati et al. 1992). This limits the sample of stars that this technique can be applied to, both in terms of the brightness and the projected rotational velocity. ZDI at the AAT thus normally targets stars of visual magnitude 9 or brighter that have $v \sin i$ in excess of $20 \mathrm{~km} \mathrm{~s}^{-1}$ (Donati et al. 1992).

This limit of $V=9$ is achieved through the use of leastsquares deconvolution (LSD; Donati et al. 1997). With the magnetic signature being very small, it is impossible to extract this information from a single spectral line. As there are several thousand photospheric lines in each échelle spectrum, the assumption is that each of these lines is affected in a similar way by the presence of spot and magnetic features in the stellar photosphere. LSD allows the extraction of the intensity (as well as the magnetic information) by combining each of the line profiles, thereby producing a single composite, high $\mathrm{S} / \mathrm{N}$ line profile. Because of the overlap associated with the orders of an échelle spectrograph, the number of lines used in the LSD extraction is more than the number of lines in the spectrum. Many of the lines appear twice and are treated as individual lines. The resulting multiplex gain obtained from LSD can be up to 40 times the $\mathrm{S} / \mathrm{N}$ obtained from a single line (Donati et al. 1997). Therefore, LSD can be used to increase the S/N in both DI and ZDI data while still retaining the intensity and polarization information in the spectrum.

To date, most stars observed using ZDI are active binaries such as RS CVn systems, with limited relevance to the Sun. Only three solar-type dwarfs have been studied in detail using ZDI: HR 1817, AB Doradus, and LQ Hydrae. AB Dor and LQ Hya are lower-mass, K-type zero-age main sequence dwarfs. AB Dor rotates extremely fast $\left(v \sin i=89 \mathrm{~km} \mathrm{~s}^{-1}, v_{\mathrm{eq}} \sim 100 \mathrm{~km} \mathrm{~s}^{-1}\right.$; Donati et al. 
2003) and LQ Hya ( $v \sin i=26 \mathrm{~km} \mathrm{~s}^{-1}, v_{\mathrm{eq}} \sim 30 \mathrm{~km} \mathrm{~s}^{-1}$; Donati et al. 2003) exhibits extreme levels of activity even though its projected rotation rate is less than one third that of AB Dor. On the other hand, HR1817 is a warm, F-type star that has a rotation period of $1 \mathrm{~d}$ that makes obtaining adequate phase coverage problematical (M. W. Mengel 2005 , in preparation). These three stars constitute a very small sample, and there remains a need to identify more nearby and bright solar-type stars with rotation rates fast enough for DI and ZDI.

Our survey targets have been chosen primarily from a southern hemisphere survey of 800 stars conducted by Henry et al. (1996). This survey determined the chromospheric emission in the cores of the CaII $\mathrm{H}$ - and $\mathrm{K}$-lines. As a result of this survey, Soderblom et al. (1998) conducted high-resolution spectroscopy of the very active stars within the original sample of 800 stars. Of the 18 most active stars selected, they found that the majority belonged to close binary systems. However, they did find five stars that were either single or well-separated binaries. Of these five stars, only PZ Tel (HD 174429) rotates fast enough for ZDI, but with a rotational period of approximately $1 \mathrm{~d}$, complete phase coverage during a single AAT run is almost impossible.

The purpose of this survey was to extend the original high-resolution spectroscopy to include stars that were not considered very active by Soderblom et al. (1998) but nevertheless still showed high levels of activity based on the $\log R_{\mathrm{HK}}^{\prime}$ indices. This survey targets stars that have not been previously observed in similar surveys (e.g. Strassmeier et al. 2000) and hence represents a new dataset.

\section{Observations and Data Analysis}

\subsection{Mt Stromlo Observations}

High-resolution spectra of 27 nearby solar-type stars were obtained over seven nights between 2001 October 4 and 10 on the Mt Stromlo Observatory 1.9-m telescope with the échelle spectrograph using the 31.6 lines $\mathrm{mm}^{-1}$ grating and a SITe $2048 \times 4096$ CCD with a 158 lines $\mathrm{mm}^{-1}$ cross disperser. The slit width was $300 \mu \mathrm{m}$, yielding a spectral resolution of $R \sim 82000$ with a dispersion of $0.008 \mathrm{~nm}$ per pixel. This was based on the measurements of the full width half maximum (FWHM) of the thorium and argon lines near the center of the spectrum. A two-times binning along the dispersion axis was used so as to reduce the readout time and the readout noise. The spectra were centered near $\mathrm{H} \alpha$ with coverage from 536 to $772 \mathrm{~nm}$. Wavelength calibration was determined using thorium-argon spectra.

\subsection{AAT Observations}

High-resolution spectra of a further 15 nearby solar-type stars were obtained over a six-night period, as a supplementary program to the DI of existing targets. The data were taken between 2002 April 27 and May 2, using the 3.9-m AAT telescope and the University College of London Échelle Spectrograph with the 31.6 lines $\mathrm{mm}^{-1}$ grating and the EEV2 detector CCD with a 31 lines $\mathrm{mm}^{-1}$ cross disperser. The slit width was $710 \mu \mathrm{m}$, yielding a spectral resolution of $R \sim 51000$ with a dispersion of approximately $0.01 \mathrm{~nm}$ per pixel. This was based on the measurements of the FWHM of the thorium-argon lines near the center of the spectrum. The spectra were centred near $526.1 \mathrm{~nm}$ with a wavelength coverage of 433.8 to $665.3 \mathrm{~nm}$. As this was a supplementary program to other AAT studies, the central wavelength and spectral coverage could not be altered to extend into the region containing lithium $670.78 \mathrm{~nm}$, as with the Mt Stromlo spectra. Wavelength calibration was determined using a thorium-argon spectrum.

\subsection{Data Analysis}

The primary aim was to determine the projected rotational velocity $(v \sin i)$ of each of the targets and to measure the emission equivalent widths of $\mathrm{H} \alpha$ (as a proxy for activity) and the equivalent width of $670.78 \mathrm{~nm}$ LiI (as an age indicator). The initial data reduction was completed using the ESPRIT software package (Donati et al. 1997). Preliminary processing involved removing the bias and flat fielding using a nightly master flat. The mean pixel resolution for the Mt Stromlo spectra was determined to be $1.529 \mathrm{~km} \mathrm{~s}^{-1}$ pixel $^{-1}$ while the mean pixel resolution for the AAT spectra was determined to be $1.689 \mathrm{~km} \mathrm{~s}^{-1}$ pixel $^{-1}$. After using ESPRIT, LSD was applied to the reduced spectra. As discussed previously, LSD combines the information from many spectral lines to produce a single line profile, thereby providing an enormous multiplex gain in the $\mathrm{S} / \mathrm{N}$.

The LSD profiles generated using the Mt Stromlo data used in excess of 600 photospheric spectral lines, and over 2800 lines were used to determine the composite line profile of the star from the AAT data. The ability to combine so many spectral lines has the advantage of increasing the $\mathrm{S} / \mathrm{N}$ many times. AAT spectra typically give an LSD profile with an equivalent $\mathrm{S} / \mathrm{N}$ in excess of 500 . For example, a $300 \mathrm{~s}$ exposure using the AAT of the G2/3V dwarf HD $62850\left(m_{\mathrm{v}}=7.83\right)$ gives a mean $\mathrm{S} / \mathrm{N}$ of almost 800 after LSD.

Along with being a useful technique for the measurement of rotational broadening (see Section 3.1), the high $\mathrm{S} / \mathrm{N}$ of the LSD profile also makes it an excellent way to determine if a star is a spectroscopic binary. A small section of the spectrum along with the corresponding LSD profile of one of these stars, HD 145523, is shown in Figure 1. There are two distinct profiles, indicating the possibility of this star being a binary system.

\section{Results and Analysis}

\subsection{Projected Rotational Velocity}

The rotational velocity of each star was measured using two techniques. The first technique utilizes a Fourier transform of the spectral line as described in detail by Gray (1992). The fast Fourier transform (FFT) of a number of spectral lines was taken and the zero of the first lobe was 

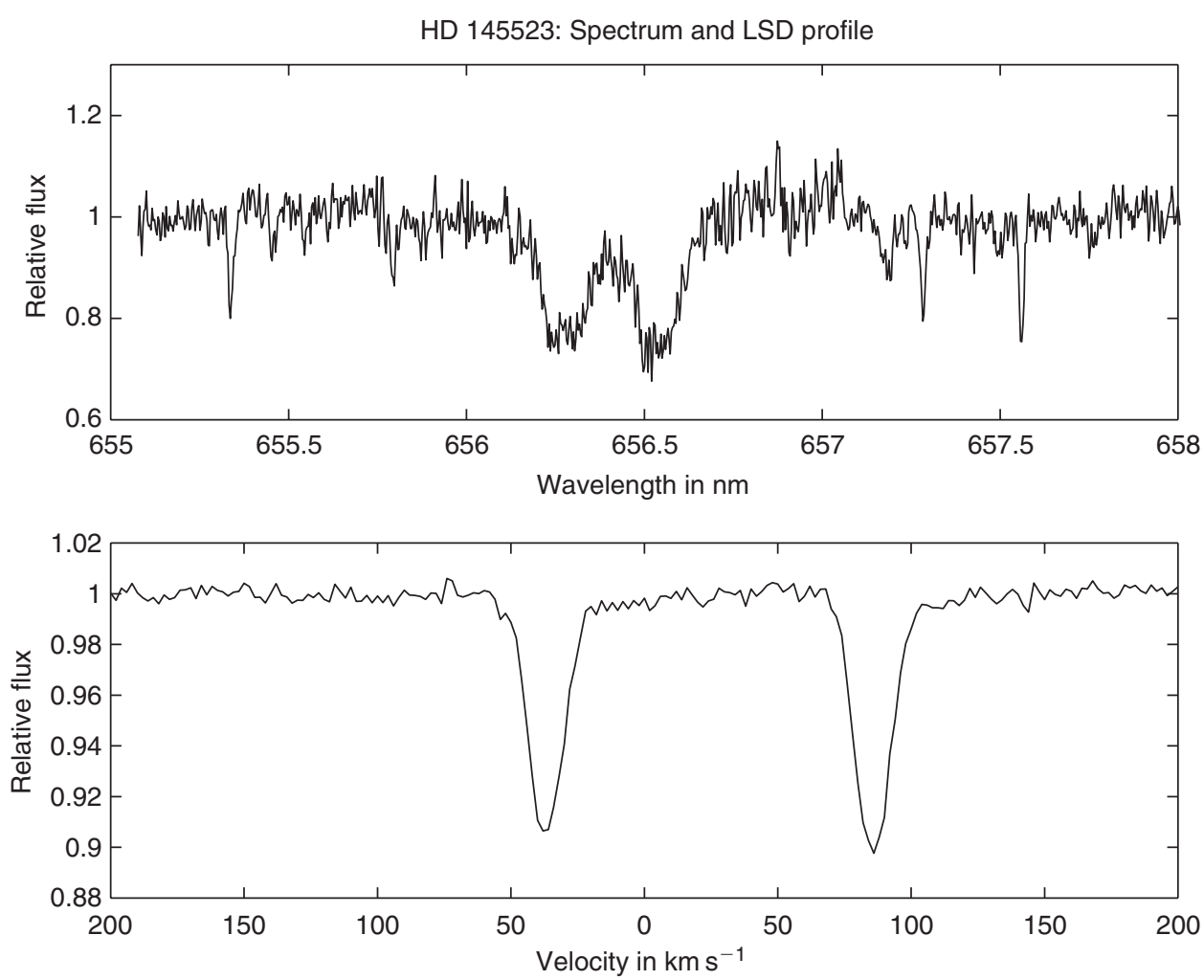

Figure 1 The top plot represents a small section of the spectrum taken at Mt Stromlo Observatory of HD 145523. The bottom plot is the LSD profile for this binary star. More than 600 individual photospheric line features have been combined to increase the S/N ratio.

calculated according to the solution given by Collins \& Truax (1995). The lines used were $492.3 \mathrm{~nm}$ FeII (Deeming 1977), $448.1 \mathrm{~nm}$ MgII, $440.5 \mathrm{~nm}$ FeI, $447.3 \mathrm{~nm}$ FeI, $447.6 \mathrm{~nm}$ Fer, $454.9 \mathrm{~nm}$ Fer, $450.8 \mathrm{~nm}$ FeII (Gray 1992), $462.39 \mathrm{~nm}$ FeII, $462.93 \mathrm{~nm}$ FeII, $461.61 \mathrm{~nm}$ CrI, $4626.1 \mathrm{~nm}$ CrI (Smith 1976), and $671.77 \mathrm{~nm}$ CaI. Not all spectral lines were used to determine $v \sin i$ for each star. A selection of lines that were identifiable and appeared free of blends was used. The $v \sin i$ for each star was then calculated as the average of the number of measurements for a number of spectral lines and the error was the standard deviation of all of the measurements. The FFT method provides a direct and independent measurement of $v \sin i$.

The second technique used for measuring $v \sin i$ involved taking the LSD profile of an inactive star of similar spectral type to the target star and rotationally broadening it to that of the target star, using the standard rotation profile described by Gray (1992). In most cases a solar spectrum was used. Due to the high S/N of the LSD profiles (from $\sim 300$ to 900 ), the fit to the rotationally broadened profile could usually be found to $\pm 1 \mathrm{~km} \mathrm{~s}^{-1}$ (Marsden 2004). To avoid the problems of rapid rotation and photospheric spot deforming the LSD profile, only the wings were used to determine the $v \sin i$. As shown by Figure 2, the results of the two techniques produce commensurate results. Nevertheless, we adopt LSD rather than FFT values for $v \sin i$ as the errors were smaller, particularly for large $v \sin i$.

Table 1 shows the projected rotational velocities, measured using the LSD profile-fitting technique, for the target

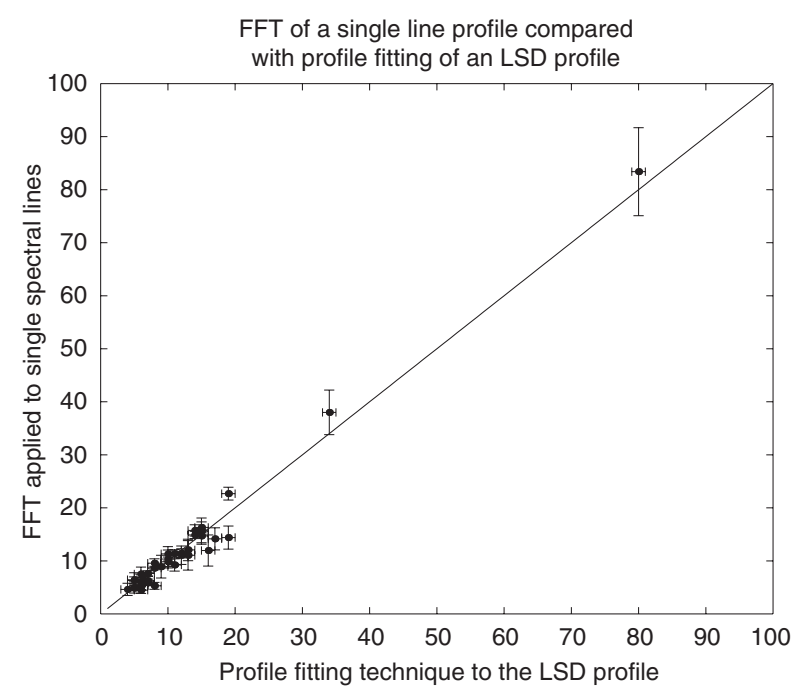

Figure 2 The relationship between the results of $v \sin i$ measurements found using an FFT of a single line profile compared with $v \sin i$ measurements found using profile fitting of the LSD profile using a rotationally broadened spectrum from a standard star of similar spectral type.

stars. Many of these $v \sin i$ values have not been previously determined.

Five of our target stars were spectroscopic binary stars. These were HD 145523, HD 75997 (although this star was not one listed by Henry et al. 1996), HD 195521, HD 202996, and HD 210395. More studies of these stars are required in order to determine the exact nature of these 
Table 1. Projected rotational velocities, radial velocities, and emission equivalent widths for the $\mathrm{H} \alpha$ line

\begin{tabular}{|c|c|c|c|c|c|c|c|}
\hline $\begin{array}{l}\text { HD } \\
\text { number }\end{array}$ & $\begin{array}{c}\text { Data } \\
\text { source }\end{array}$ & $\begin{array}{l}\text { Spectral } \\
\text { type }\end{array}$ & $\begin{array}{l}\text { Number } \\
\text { of spectra }\end{array}$ & $\begin{array}{c}v \sin i \\
{\left[\mathrm{~km} \mathrm{~s}^{-1}\right]^{\mathrm{B}}}\end{array}$ & $\begin{array}{c}\text { Radial vel. } \\
{\left[\mathrm{km} \mathrm{s}^{-1}\right]}\end{array}$ & $\begin{array}{c}\text { EEW H } \alpha \\
{[\mathrm{m} \AA]}\end{array}$ & $\begin{array}{c}\text { Error in EEW } \\
{[\mathrm{m} \AA]}\end{array}$ \\
\hline 105 & (a) & G0V & 4 & 14 & 0.7 & 137 & 14 \\
\hline 1237 & (a) & G6V & 4 & 8 & -6.8 & 75 & 15 \\
\hline 1466 & (a) & F8/G0V & 4 & 19 & 6.2 & 109 & 18 \\
\hline 18809 & (a) & G3V & 3 & 10 & 7.1 & 124 & 26 \\
\hline 36435 & (a) & G6/G8V & 2 & 5 & 15.6 & 60 & 9 \\
\hline \multirow[t]{2}{*}{38397} & (a) & G0V & 2 & 15 & 24.4 & & \\
\hline & (b) & G0V & 1 & 15 & 24 & 148 & 2 \\
\hline 59967 & (a) & G4V & 1 & $\sim 5$ & 8.8 & 10 & 1 \\
\hline 61005 & (b) & G3/G5V & 1 & 8 & 23 & 136 & 6 \\
\hline 61033 & (b) & G5/6 V & 1 & $<6$ & 24 & 181 & 25 \\
\hline 62848 & (b) & G0V & 1 & 12 & 22 & 72 & 8 \\
\hline \multirow[t]{2}{*}{62850} & (a) & $\mathrm{G} 2 / 3 \mathrm{~V}$ & 1 & 15 & 16.4 & 97 & 19 \\
\hline & (b) & $\mathrm{G} 2 / 3 \mathrm{~V}$ & 1 & 14 & 18 & 74 & 4 \\
\hline 72687 & (b) & G5V & 1 & 7 & 22 & 197 & 28 \\
\hline 74576 & (b) & K1V & 1 & $<6$ & 14 & 90 & 9 \\
\hline 75519 & (b) & G5V & 1 & 8 & 22 & 144 & 24 \\
\hline 84273 & (b) & G5V & 1 & $\sim 6$ & 13 & 175 & 25 \\
\hline 90712 & (b) & $\mathrm{G} 2 / 3 \mathrm{~V}$ & 1 & 11 & 20 & 103 & 31 \\
\hline 105690 & (b) & G5V & 1 & 9 & 17 & 234 & 43 \\
\hline 106506 & (b) & F8/G0V & 1 & 80 & 10 & 534 & 39 \\
\hline 113553 & (b) & G5V & 1 & 10 & 2 & 194 & 42 \\
\hline 118972 & (b) & $\mathrm{K} 2 \mathrm{~V}$ & 1 & $<6$ & -6.0 & 90 & 6 \\
\hline 141943 & (b) & G1V & 2 & 34 & -1.5 & 455 & 20 \\
\hline 150689 & (a) & $\mathrm{K} 2 \mathrm{~V}$ & 2 & 6 & -20.1 & 207 & 92 \\
\hline 152391 & (a) & G8V & 2 & $\sim 5$ & 43.2 & 39 & 8 \\
\hline 165185 & (a) & G5V & 3 & 8 & 14.2 & 58 & 13 \\
\hline 181321 & (a) & G5V & 3 & 13 & -10.8 & 142 & 15 \\
\hline 183414 & (a) & G3V & 4 & 10 & 2.3 & 133 & 19 \\
\hline 190422 & (a) & F8V & 3 & 19 & 12.1 & 67 & 20 \\
\hline 193464 & (a) & G0/G1V & 2 & 17 & -29.5 & 64 & 14 \\
\hline 202732 & (a) & G5V & 2 & $\sim 5$ & -2.8 & 99 & 21 \\
\hline 203019 & (a) & G5V & 2 & 7 & 23.9 & 103 & 14 \\
\hline 203244 & (a) & G5V & 3 & $<5$ & 19.7 & 107 & 15 \\
\hline 209234 & (a) & G3V & 2 & 13 & 8.5 & 111 & 13 \\
\hline 215657 & (a) & G3V & 2 & 11 & 10.5 & 89 & 19 \\
\hline 215768 & (a) & G0V & 3 & 7 & -26.5 & 119 & 14 \\
\hline 217343 & (a) & G3V & 4 & 12 & 5.8 & 139 & 14 \\
\hline 223537 & (a) & G3V & 2 & 16 & 23.1 & 176 & 20 \\
\hline
\end{tabular}

A The data were obtained using (a) the 1.9-m Mt Stromlo telescope and (b) the 3.9-m AAT.

${ }^{\mathrm{B}}$ Derived from the LSD profile.

systems. Applying the LSD profile-fitting technique to the two components of HD 195521 produced $v \sin i$ values of $\sim 5-6 \mathrm{~km} \mathrm{~s}^{-1}$. We note that HD 195521 also has an observed variability of $0.024 \mathrm{mag}$ in $V$-band photometry (HIPPARCOS) and is listed as an IRAS source. LSD profile fitting for the components of HD 145523 produced $v \sin i$ values of 6 and $<5 \mathrm{~km} \mathrm{~s}^{-1}$. The two components in HD 210395 have $v \sin i$ values of 6 and $<5 \mathrm{~km} \mathrm{~s}^{-1}$. One of the components of HD 202996 has a $v \sin i$ of $6 \mathrm{~km} \mathrm{~s}^{-1}$ while the other component was too small to be determined. The two components of the LSD profile of HD 75997 were not sufficiently resolved to allow for the determination of individual $v \sin i$ values.

\subsection{Heliocentric Radial Velocity}

The LSD profile also can be used to measure the radial velocity of the star. The observed velocity shift of the
LSD profile was converted to heliocentric radial velocity using RVCORRECT in IRAF, using the usual parameters for solar motion. The resulting radial velocities are listed in Table 1. When comparing the results of this survey with the larger survey by Montes et al. (2001a), there were six stars in common. There was general agreement, within their respective errors, between the radial velocities measured for these stars.

\subsection{Ha Activity: A Proxy for Stellar Activity}

The $\mathrm{H} \alpha$ line was used as a proxy for stellar activity. Thatcher \& Robinson (1993) suggest that in the presence of an active chromosphere, the $\mathrm{H} \alpha$ line is collisionally filled in as a result of the higher temperatures within the lower levels of the chromosphere. The increased temperature of an active chromosphere has the effect of increasing 
Difference spectra of HD 62850, compared with a rotationally broadened solar spectrum

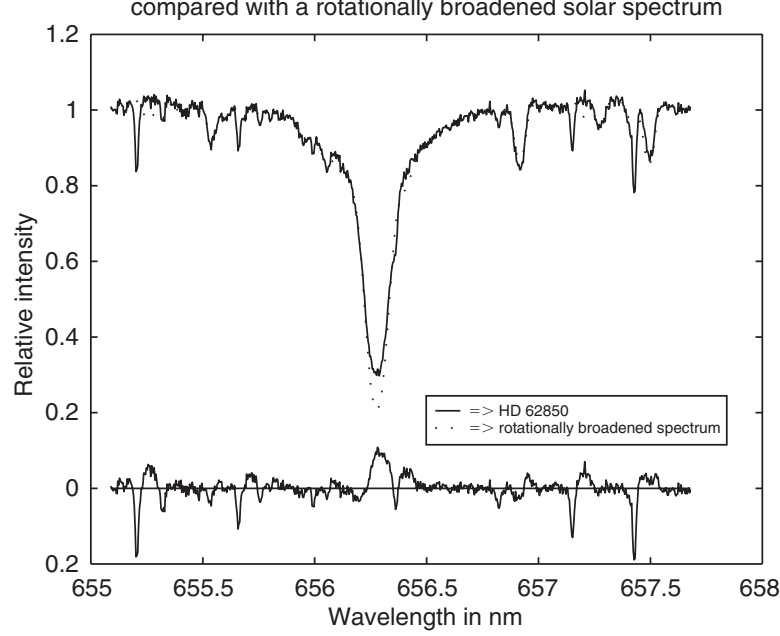

Figure 3 The $\mathrm{H} \alpha$ emission of HD 62850. The upper plot is a spectrum of the star prior to the subtraction of a rotationally broadened spectrum of a low-activity star (i.e. the solar spectrum). The lower plot shows the resulting net $\mathrm{H} \alpha$ emission for HD 62850 used to measure its EEW and demonstrating the filling-in of the photospheric line by chromospheric activity.

the electron density at this level where the $\mathrm{H} \alpha$ line is formed.

Thatcher \& Robinson (1993) suggested that the equivalent width of the $\mathrm{H} \alpha$ line is not a good measure of activity. Instead, many authors such as Soderblom et al. (1993a) determine the emission component of the $\mathrm{H} \alpha$ line by subtracting the spectrum of a typical standard star with a similar spectral type, normally the solar spectrum. The resulting emission equivalent width (EEW) is a measure of the active chromosphere component of the $\mathrm{H} \alpha$ line.

An example of this subtraction process is shown in Figure 3 where the spectrum near the $\mathrm{H} \alpha$ line of $\mathrm{HD}$ $62850(\mathrm{G} 2 / 3 \mathrm{~V})$ is subtracted from a rotationally broadened solar spectrum $(\mathrm{G} 2 \mathrm{~V})$. The resulting EEW of the difference spectrum was determined by finding the area under the line profile. The final EEW measurement for each star was the average of a number of measurements taken and using a number of non-active standard stars. Apart from solar spectra, non-active standard stars used to determine the EEW for the Mt Stromlo data were HD 11131 (G0V), HD 38973 (G2V), HD 158614 (G9IV/V), and HD $202628(\mathrm{G} 2 \mathrm{~V})$. The non-active standard stars used to determine the EEW for the AAT data were HD 55720 (G8V), HD 90170 (G8IV/V), HD 73256 (G8/K0V), HD 128621 (K1V), and HD 126821 (K3III).

Stellar models by Kurucz (1993) were used to transform the EEW into a flux. In order to investigate the relationship between rotational velocity and activity, the Rossby number (Stępień 1994) was calculated for each star. The Rossby number is the ratio of the rotation period $P_{\text {rot }}$ to the theoretically determined turnover time for the bottom layers of the convection zone (Noyes et al. 1984; Montesinos et al. 2001). Many of the stars in this survey have no published photometric period so an approximation

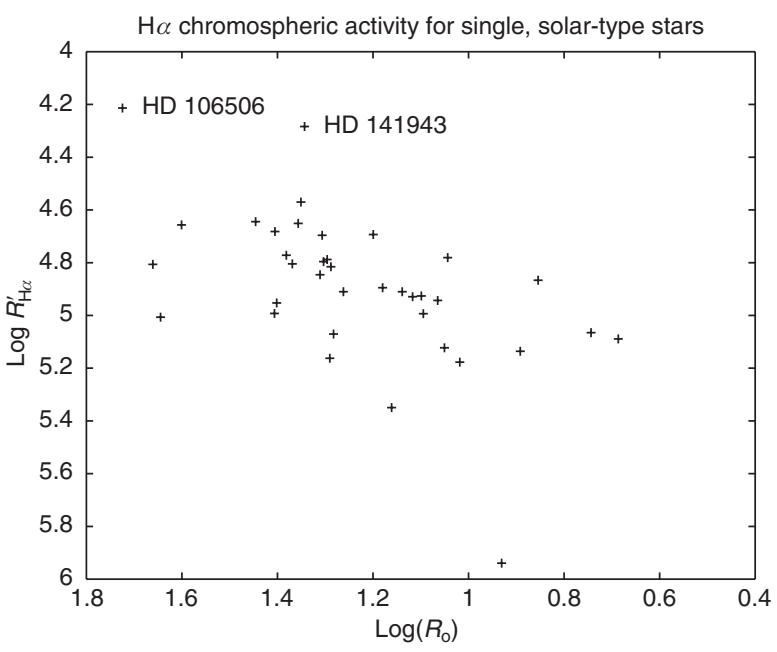

Figure 4 A $\log -\log$ plot of the relative flux $\left(R_{\mathrm{H} \alpha}^{\prime}\right)$ as a function of Rossby number $\left(N_{\mathrm{R}}^{\prime}\right)$ for the sample of solar-type stars observed.

to the Rossby number for all stars was used (Soderblom et al. 1993a):

$$
N_{\mathrm{R}}^{\prime}=2 \pi R_{\mathrm{o}} / \tau_{\mathrm{c}} v \sin i
$$

where $R_{\mathrm{O}}$ is the estimate of the radius of the star, based on the bolometric corrections of Bessell et al. (1998), and $\tau_{\mathrm{c}}$ is the theoretical convective turnover time, estimated from $(B-V)_{\mathrm{o}}$ using the equations in Noyes et al. (1984). In effect, this is an upper limit to the true Rossby number $N_{\mathrm{R}}$ as $v \sin i \leq v_{\text {eq }}$. The resulting $\log -\log$ graph of the activity of each star is shown in Figure 4.

\subsection{Lithium 670.78-nm Line-An Age Indicator}

The $670.78 \mathrm{~nm}$ LiI line is often used as an indicator of youth for single stars. The equivalent width of the $670.78 \mathrm{~nm}$ LiI line was determined for each star. Stars with a rotational velocity in excess of $8 \mathrm{~km} \mathrm{~s}^{-1}$ often have the weak 670.74-nm Fer line blended with the Li line. The equivalent width for the Li line was corrected for the presence of the nearby iron line using the same correction factor developed by Soderblom et al. (1993b, 1993c). The corrected equivalent width was compared with the 671.77-nm CaI line in order to determine the youthful nature of the star. The equivalent width of the Li line was used in conjunction with the analysis of equivalent widths versus spectral type by Montes et al. (2001b). From this, an approximate age for some of these stars was determined. Table 2 shows the age estimate for the stars that have the Li feature in the spectrum. Figure 5 shows the strength of the lithium feature, as compared with the 671.77-nm CaI line for the stars with the strongest feature, as observed at Mt Stromlo.

\section{Comments on Some Individual Stars}

HD 1466 is an F8/G0 V star. It has a projected rotational velocity of $19 \mathrm{~km} \mathrm{~s}^{-1}$. It has a temperature of approximately $6130 \mathrm{~K}$, estimated on the basis of its $V-I$ colour index using the bolometric corrections of Bessell et al. 
Table 2. A comparison between LiI $670.78 \mathrm{~nm}$ and CaI $671.7 \mathrm{~nm}$

\begin{tabular}{|c|c|c|c|c|}
\hline $\begin{array}{l}\text { HD } \\
\text { number }\end{array}$ & $\begin{array}{l}\text { Spectral } \\
\text { type }\end{array}$ & $\begin{array}{c}\text { EEW } \\
\text { CaI [mA] }\end{array}$ & $\begin{array}{c}\text { Corrected EEW } \\
\text { LiI }[\mathrm{m} \AA]\end{array}$ & Comments ${ }^{\mathrm{A}}$ \\
\hline 105 & G0V & 99.28 & 147.1 & Pleiades age \\
\hline 1237 & G6V & 146.3 & 44.11 & older than Hyades age \\
\hline 1466 & $\mathrm{~F} 8 / \mathrm{G} 0 \mathrm{~V}$ & 97.79 & 128.9 & Pleiades age \\
\hline 18809 & G3V & 97.07 & 98.9 & approaching Hyades age \\
\hline 38397 & G0V & 93.12 & 158.2 & Pleiades age \\
\hline 62850 & $\mathrm{G} 2 / 3 \mathrm{~V}$ & 188.5 & 127.2 & Pleiades age \\
\hline 165185 & G5V & 112.8 & 76.4 & Hyades age \\
\hline 181321 & G5V & 112.5 & 110.5 & between Pleiades and Hyades age \\
\hline 183414 & G3V & 105.5 & 134.14 & near Pleiades age \\
\hline 190422 & F8V & 110.1 & 105.94 & Pleiades age \\
\hline 193464 & G0/G1V & 149.5 & 91.44 & Hyades age \\
\hline 202732 & G5V & 102.2 & 7.65 & \\
\hline 203244 & G5V & 123.5 & 1.62 & \\
\hline 209234 & G3V & 117.1 & 84.52 & Hyades age \\
\hline 215657 & G3V & 116.5 & 70.62 & Hyades age \\
\hline 215768 & G0V & 83.45 & 59.73 & \\
\hline 217343 & G3V & 112.1 & 163.5 & Pleiades age \\
\hline
\end{tabular}

${ }^{\mathrm{A}}$ Age estimates based on the equivalent width of the lithium line using Figure 2 from Montes et al. (2001b) as a reference figure.

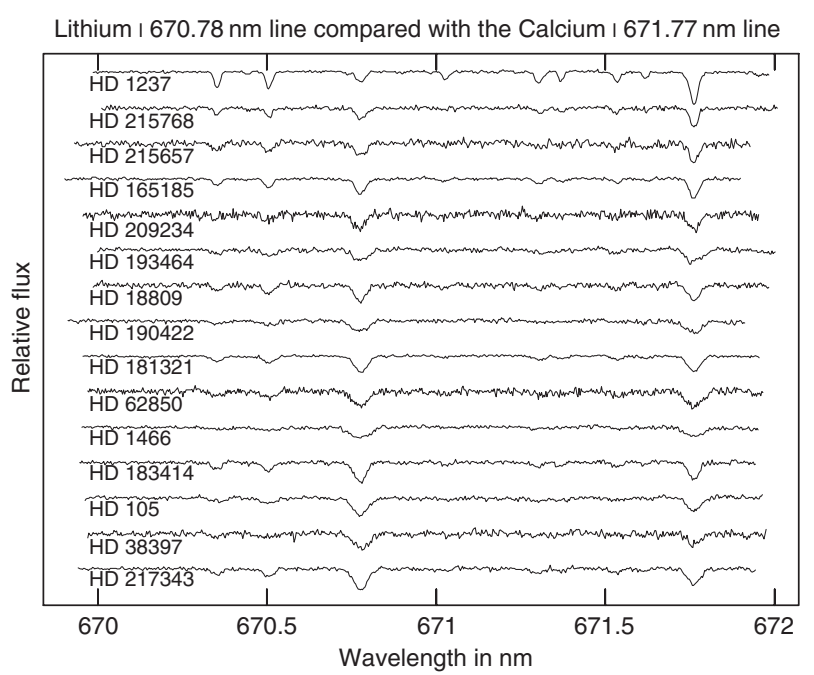

Figure 5 The spectra of 15 young stars in the sample, as judged by their strong Lir 670.78-nm line. The CaI 671.77-nm feature is shown for comparison.

(1998). HD 1466 showed evidence of spots in the line profiles on two of the spectra taken on 2001 October 5, but the spots were not as obvious on the other two spectra taken on 2001 October 9. This star is relatively nearby at a distance of $40.95 \mathrm{pc}$ (HIPPARCOS) and hence is relatively bright at an apparent magnitude of 7.47 (SIMBAD). It was found to have limited chromospheric activity with an EEW of $109 \pm 18 \mathrm{~m} \AA$, giving $\log R_{\mathrm{H} \alpha}^{\prime} \sim-4.9$. Because of the modest $v \sin i$, this star makes a possible but not ideal target for DI/ZDI.

HD 106506 is an F8/G0V star. It has a trigonometric parallax of 7.96 milliarcseconds (mas) giving a distance of approximately $126 \mathrm{pc}$ (HIPPARCOS). It has an apparent visual magnitude of 8.49 (SIMBAD). The LSD line

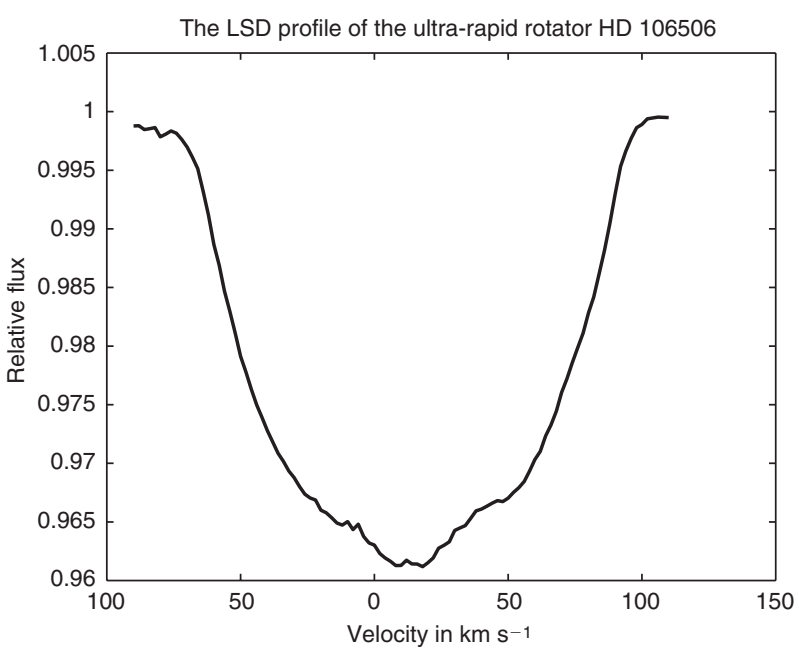

Figure 6 The LSD profile for HD 106506, an active, young, apparently single star with very rapid rotation $\left(v \sin i=80 \mathrm{~km} \mathrm{~s}^{-1}\right)$. The distortion of the profile near zero velocity is suggestive of spot features on the stellar surface.

profile, as shown in Figure 6, shows evidence of spot activity on the photosphere, with perhaps a giant polar spot being observed. The deformation of the line profile is an indicator of these features. There was no evidence in the line profiles to indicate that this activity was as a result of a binary system, unlike the deduction by Soderblom et al. (1998). (They concluded that HD 106506 could be a possible double-lined spectroscopic binary - SB2 star.) The spectra obtained by the 3.9-m AAT, coupled with LSD, gives sufficient resolution to conclude that there is no evidence of HD 106506 being a binary star system. This supports the conclusion by Mason et al. (1998) and Cutispoto et al. (2002) that HD 106506 is a single star. 
There is evidence of an active chromosphere with the $\mathrm{H} \alpha$ line collisionally filled in, as demonstrated in the strength of the EEW of $534 \pm 39 \mathrm{~m} \AA$, giving $\log R_{\mathrm{H} \alpha}^{\prime} \sim-4.21$. HD 106506 is a very rapidly rotating star. Soderblom et al. (1998) measured a projected rotational velocity of $70 \pm 8 \mathrm{~km} \mathrm{~s}^{-1}$ and Cutispoto et al. (2002) measured a projected rotational velocity of approximately $125 \mathrm{~km} \mathrm{~s}^{-1}$. The two $v \sin i$ values are discrepant and we have used the star's LSD profile to measure a projected rotational velocity of $80 \mathrm{~km} \mathrm{~s}^{-1}$ and FFTs of individual spectral lines to give a $v \sin i$ of $83.4 \pm 8.3 \mathrm{~km} \mathrm{~s}^{-1}$. We adopt the LSD value over the slightly higher FFT result as we believe it to be a more robust measurement for active stars. The ultra-fast rotation of this star, coupled with its strong chromospheric activity, makes it an excellent potential target for follow-up magnetic studies using DI and ZDI.

HD 141943 is another suitable candidate for followup DI/ZDI studies. It has a parallax of 7.5 mas giving a distance of approximately $133 \mathrm{pc}$ (HIPPARCOS). It has an apparent visual magnitude of 7.87 (SIMBAD). This G1V star has a projected rotational velocity of $34 \mathrm{~km} \mathrm{~s}^{-1}$. There is evidence of an active chromosphere with an EEW of $455 \pm 20 \mathrm{~m} \AA$, giving $\log R_{\mathrm{H} \alpha}^{\prime} \sim-4.28$. There was no evidence in the LSD line profile to indicate that this activity was as a result of a binary system. We deduce that the resulting activity is related to the rotational speed of this single star. Although we did not collect LiI 671.78-nm data for an age estimate for HD 141943, this star is regarded as a pre-main sequence object by Cutispoto et al. (2003). The LSD profile gave no indication of the presence of large spot features. Given the activity and the rapid rotation, further observations of this star are warranted to see if other rotational phases show spot features.

\section{Conclusions}

The primary aim of this work was to identify active, young, single, Sun-like dwarfs that rotate fast enough for mapping of their starspots and surface magnetic fields. Based on our $\mathrm{H} \alpha$ activity analysis and $v \sin i$ measurements, the two most promising targets are HD 106506 (F8V/G0V) and HD 141943 (G1V). Of these two, the better candidate seems to be HD 106506, due to its high activity, rapid rotation, and indications that is young and single. Although both HD 106506 and HD 141943 may serve as young solar proxies, precision photometry of starspot variability is required to confirm the presence of major spot features, as well as ensure their rotation periods are not close to one day and hence compatible with phase coverage requirements for DI and ZDI.

\section{Acknowledgments}

We would like to thank Professor Mike Bessell for his first night assistance on the 1.9-m Mt Stromlo telescope. We also appreciate the assistance of the technical staff at both RSAA and AAO for their expertise and cheerful assistance. May the memory of the 1.9-m Mt Stromlo telescope live on well into the 21 st century. We appreciate the support of Dr Jean-François Donati for supplying us his ESPRIT code and to Dr Rhodes Hart for his assistance and expertise in programming. We would like to thank the anonymous referee for the time taken over this paper and for the suggestions on improving the quality of this work. This survey was made possible with a Team Project Grant from the University of Southern Queensland.

\section{References}

Bessell, M. S., Castelli, F., \& Plez, B. 1998, A\&A, 333, 231

Collins II, G. W., \& Truax, R. J. 1995, ApJ, 439, 860

Cutispoto, G., Pastori, L., Pasquini, L., de Medeiros, J. R., Tagliaferri, G., \& Andersen, J. 2002, A\&A, 384, 491

Cutispoto, G., Tagliaferri, G., de Medeiros, J. R., Pastori, L., Pasquini, L., \& Andersen, J. 2003, A\&A, 397, 987

Deeming, T. J. 1977, Ap\&SS, 46, 13

Donati, J.-F., Semel, M., \& Praderie, F. 1989, A\&A, 225, 467

Donati, J.-F., Semel, M., \& Rees, D. E. 1992, A\&A, 265, 669

Donati, J.-F., Semel, M., Carter, B. D., Rees, D. E., \& Cameron, A. C. 1997, MNRAS, 291, 658

Donati, J.-F., et al. 2003, MNRAS, 345, 1145

Fekel, F. 1980, BAAS, 12, 500

Gray, D. F. 1992, The Observation and Analysis of Stellar Photospheres, 2nd edn (Cambridge: Cambridge University Press), 392

Henry, T. J., Soderblom, D. R., Donahue, R. A., \& Baliunas, S. L. 1996, AJ, 111, 439

Kurucz, R. L. 1993, CDROM \#13 (ATLAS9 Atmospheric Models) and \#18 (ATLAS9 and SYNTHE routines) spectral line databases

Marsden, S. C. 2004, PhD Thesis (Toowomba: University of Southern Queensland)

Marsden, S. C., Waite, I. A., Carter, B. D., \& Donati, J.-F. 2004, AN, 325,246

Mason, B. D., Henry, T. J., Hartkopf, W. I., Ten Brummelaar, T., \& Soderblom, D. R. 1998, AJ, 116, 2975

Montes, D., López-Santiago, J., Gálvez, M. C., FernándezFigueroa, M. J., De Castro, E., \& Cornide, M. 2001a, MNRAS, 328,45

Montes, D., López-Santiago, J., Fernández-Figueroa, M. J., \& Gálvez, M. C. 2001b, A\&A, 379, 976

Montesinos, B., Thomas, J. H., Ventura, P., \& Mazzitelli, I. 2001, MNRAS, 326, 877

Noyes, R. W., Hartmann, L. W., Baliunas, S. L., Duncan, D. K., \& Vaughan, A. H. 1984, ApJ, 279, 763

Semel, M. 1989, A\&A, 225, 456

Semel, M., Donati, J.-F., \& Rees, D. E. 1993, A\&A, 278, 231

Smith, M. A. 1976, ApJ, 208, 487

Soderblom, D. R., King, J. R., \& Henry, T. J. 1998, AJ, 116, 396

Soderblom, D. R., Stauffer, J. R., Hudon, J. D., \& Jones, B. F. 1993a, ApJSS, 85, 315

Soderblom, D. R., Pilachowski, C. A., Fedele, S. B., \& Jones, B. F. 1993b, AJ, 105, 2299

Soderblom, D. R., Jones, B. F., Balachandran, S., Stauffer, J. R., Duncan, D. K., Fedele, S. B., \& Hudon, J. D. 1993c, AJ, 106, 1059

Stępień, K. 1994, A\&A, 292, 191

Strassmeier, K. G., \& Rice, J. B. 1998, A\&A, 330, 685

Strassmeier, K. G., Washuettl, A., Granzer, Th., Scheck, M., \& Weber, M. 2000, A\&AS, 142, 275

Thatcher, J. D., \& Robinson, R. D. 1993, MNRAS, 262, 1

Vogt, S. S., \& Penrod, G. D. 1983, PASP, 95, 565 